\title{
Experimental Investigation of Optoelectronic receiver with Reservoir Computing in Short Reach Optical Fiber Communications
}

\author{
Ranzini, Stenio M.; Dischler, Roman; Da Ros, Francesco; Buelow, Henning; Zibar, Darko
}

Published in:

Journal of Lightwave Technology

Link to article, DOI:

10.1109/JLT.2021.3049473

Publication date:

2021

Document Version

Peer reviewed version

Link back to DTU Orbit

Citation (APA):

Ranzini, S. M., Dischler, R., Da Ros, F., Buelow, H., \& Zibar, D. (2021). Experimental Investigation of Optoelectronic receiver with Reservoir Computing in Short Reach Optical Fiber Communications. Journal of Lightwave Technology, 39(8), 2460-2467. https://doi.org/10.1109/JLT.2021.3049473

\section{General rights}

Copyright and moral rights for the publications made accessible in the public portal are retained by the authors and/or other copyright owners and it is a condition of accessing publications that users recognise and abide by the legal requirements associated with these rights.

- Users may download and print one copy of any publication from the public portal for the purpose of private study or research.

- You may not further distribute the material or use it for any profit-making activity or commercial gain

- You may freely distribute the URL identifying the publication in the public portal 


\title{
Experimental Investigation of Optoelectronic receiver with Reservoir Computing in Short Reach Optical Fiber Communications
}

\author{
Stenio M. Ranzini, Roman Dischler, Francesco Da Ros, Henning Bülow, Darko Zibar
}

\begin{abstract}
The cloud edge data center will enable reliable and low latency options for the network, and the interconnection among these data-centers will demand a scalable lowcomplexity scheme. An intensity-modulated and directed detected transmission system is an attractive solution, but chromatic dispersion is the main limitation for higher symbol rate systems. To overcome this challenge, we have proposed and experimentally demonstrated a receiver with shared-complexity between optical and digital domains that enables $80 \mathrm{~km}$ transmission reach below KP4 FEC limit for a 32 GBd on-off keying signal. The optical stage consists of optical filters that slices the signal into smaller sub-bands and each is detected by a photodetector. A feedforward neural network and reservoir computing are compared to reconstruct the full signal from the slices and mitigate the chromatic dispersion. Both equalizers have shown similar performance with the advantage of the reservoir computing requiring fewer inputs and easier training process. In this work, we have compared the linear and nonlinear activation functions in the feedforward neural network to investigate the gain of using a nonlinear equalizer. The maximum transmission reach is reduced almost to half, $\approx 45 \mathrm{~km}$, when using the linear. The performance is also reduced if a reduced number of slices is used in the receiver, as we have demonstrated. In this case, using 2 slices to reduce the complexity of the system, instead of the total 4 , we have shown $a \approx 55 \mathrm{~km}$ transmission reach below KP4 FEC limit. In this work we have also provided a numerical comparison with $4 \times 8$ GBd subcarriers system. The results have shown a $40 \mathrm{~km}$ increase in transmission reach compared to the proposed optoelectronic system. The trade-off between performance and complexity should be analyzed for each case, as a different hardware is required in each situation.
\end{abstract}

Index Terms-Short-reach transmission, direct-detection, reservoir computing, signal equalization, chromatic dispersion.

\section{INTRODUCTION}

$\mathbf{T}$ HE upcoming 5G technology will provide a huge number of applications by connecting not only humans but also machines. This new scenario, where machine-to-machine communication will happen on a large scale, will require lowlatency services that are not able to be delivered in the current network, and this is essential to provide reliability and security. As part of the solution, traditional data-centers are moving some of the services closer to the end-user, creating the cloud

Stenio M. Ranzini, Francesco Da Ros and Darko Zibar are with the Department of Fotonik, Technical University of Denmark (DTU), Denmark, e-mail:smara@fotonik.dtu.dk

Roman Dischler and Henning Bülow are with the Optical Transmission Group, Nokia Bell Labs Stuttgat, Germany.

Manuscript received April 19, 2005; revised August 26, 2015. edge. The inter-data center communication will require a lowcomplexity system to satisfy such demands.

Coherent receivers are an attractive solution due to access to the full optical field and are well-known in long-haul systems. Nonetheless, the traditional coherent receivers are complex and power-hungry, and alternatives to simplify their structure are being investigated [1].

Intensity-modulated and direct detected (IM/DD) systems can provide low-complexity and low-latency links. However, chromatic dispersion (CD) is a major constraint to extending the transmission reach and rate in the C-band, which makes scalability a challenge. Although transmission in the O-band is possible (avoiding the intersymbol interference from $\mathrm{CD}$ ) it suffers from high attenuation [2]. Alternative techniques to mitigate the $\mathrm{CD}$ on $\mathrm{C}$-band IM/DD systems are available by acting in the optical domain [3], [4], in the digital/electric domain [5], [6], [7], [8], [9], [10], [11], [12], [13], [14], or by considering a joint optoelectronic approach [15], [16], [17], [18], [19], [20].

Optical domain techniques, such as dispersioncompensating fibers (DCFs) or fiber Bragg gratings (FBGs) mitigates all the $\mathrm{CD}$ prior-detection. However, they have a large footprint and latency (mainly DCFs), besides the significant power loss.

In the electric domain, several digital signal processing (DSP)-based techniques have already been proposed, such as maximum likelihood sequence estimation, decision feedback equalizers, and Volterra [5], [6], [7], [8]. Furthermore, DSPbased pre-distortion, such as Tomlinson Harashima precoding and the Kramers-Kronig receiver has also been demonstrated [9], [10]. Promising results have been shown by using these methods and the trade-off between performance and complexity is being discussed. Additionally, further signal processing technology for this application range has been suggested, such as DSP-implemented neural networks (NN) [11], [12], [13], [14]. However, the NN requires a training stage to correctly equalize the channel, and this process can be time and energy consuming.

An alternative solution to mitigate CD on IM/DD systems is moving to hybrid optoelectronic approaches, which leverage on sharing the complexity between optical and electrical domains. In this direction, a few recent investigations have proposed the use of silicon photonics-based reservoir computing (RC) together with analog electronic processing [16], [17], [18]. The RC has the advantage of an easy training stage, which has benefits for photonic applications. In [19], we have 


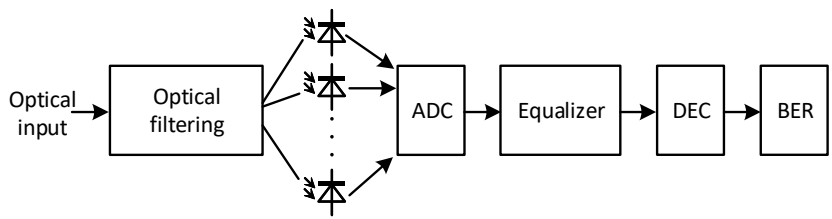

Fig. 1. Optoelectronic receiver. An optical filter splits the signal into small sub-bands and each is detected by a photodetector. The signal is then digitized by a ADC, equalized, decided (DEC) and the BER analyzed.

proposed a hybrid optoelectronic approach that consists of passive optical filters and an additional digital equalizer. More recently, we have proposed the $\mathrm{RC}$ as the digital equalizer to reduce the complexity of the training phase of the hybrid optoelectronic system [20].

In this work, we have extended our experimental results of the hybrid optoelectronic approach of [21]. Beyond the state-of-art of [21], we have compared the traditional NN equalizer with hyperbolic tangent as an activation function with a linear alternative, probing the difference of using a linear and nonlinear equalizer. The results have shown that by using the nonlinear activation function the transmission reach is almost doubled $(80 \mathrm{~km})$. We also have numerically compared the optoelectronic receiver performance with a single carrier $32 \mathrm{GBd}$ on-off keying (OOK) transmission to the subcarrier multiplexing (SCM) system with four carriers at 8 GBd OOK. The analyses have shown that the SCM system has $40 \mathrm{~km}$ transmission reach improvement compared to the hybrid optoelectronic receiver. However, at the transmitter, this scheme requires an IQ modulator for the digital subcarrier or multiple MZM in the case of the optical subcarrier. In the receiver, it also requires sharper filters to split the subcarriers or larger guardband, which decreases the spectral efficiency of the transmission. Therefore, complexity and performance will depend on the number of subcarriers that will be transmitted.

This paper is organized as follows. In Section II we review the optoelectronic receiver introduced in [19]. In Section III, we review some of the concepts of RC and the implemented algorithm is described. The Section IV and VI details the experimental and numerical setup, respectively. Section V shows the experimental results related to the optoelectronic receiver. Section VII compares numerically the optoelectronic receiver with the SCM system. Finally, Section VIII summarize the main conclusions of the paper.

\section{Optoelectronic RECEIVER}

Fig. 1 shows the optoelectronic receiver. The input signal in the receiver is sliced with optical filters and the signal of the slices is jointly processed in the equalizer. In other words, multiple filters are shifted in frequency with reduced bandwidth, covering the whole signal's bandwidth. The performance of the receiver to mitigate the $\mathrm{CD}$ in $\mathrm{DD}$ systems will depend on the number of filters, bandwidth, and relative position of each filter. Decreasing the bandwidth of each filter decreases the memory requirement to mitigate the $\mathrm{CD}$. In turn, a higher number of filters might be necessary to cover all the signal's spectrum. In [19], [20], we have numerically analyzed the

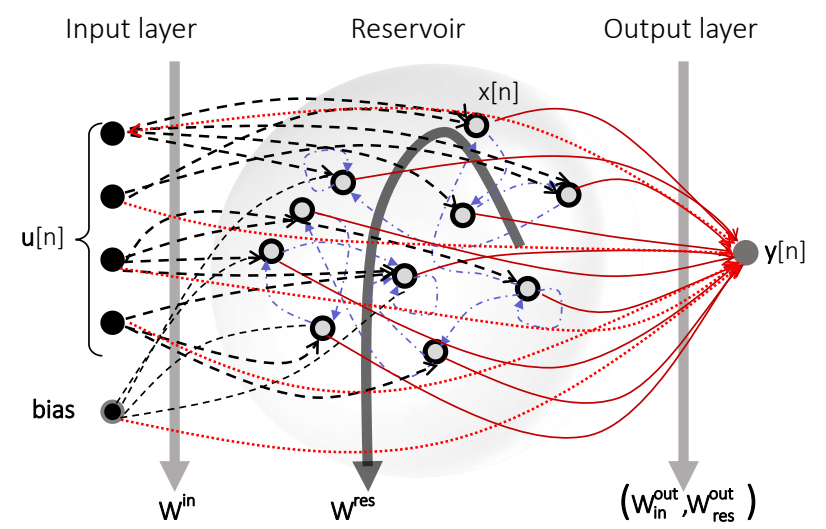

Fig. 2. Reservoir computing schematic. The red curves (full and dashed lines) indicates the weights that goes through the training process - typically a linear regression.

options of using Mach-Zehnder delay interferometers (MZDIs) and arbitrary waveguide grating (AWG) in this stage. In the case of the MZDI, each component creates 2 slices. The bandwidth and relative position of each other is described in [19]. An increasing number of slices can be achieved by concatenating a series of cascaded MZDIs. The AWG was modeled as a set of second-order Gaussian filters with a $3-\mathrm{dB}$ bandwidth of each filter, which is also equal to the frequency separation between two neighboring output filter. We have shown that the improvement for decreasing the filter's bandwidth reaches a minimum at $\approx 16 \mathrm{GHz}$ to $20 \mathrm{GHz}$ for a $32 \mathrm{GBd}$ OOK with root-raised-cosine (RRC) filter (rolloff equal to 0.1). Reducing further the bandwidth showed significant degradation. After the filtering stage, each slice is then detected by a PD. The number of PDs depends on the number of slices. The impact of using less PDs than the total available in the receiver is analyzed in Section V. After the PD, the signal is then digitized by an analog-to-digital converter (ADC) and fed to an equalizer, which primarily fulfills two tasks. The first is to reconstruct the information obtained from the sliced power spectrum and the second is to mitigate CD. Its output is then decided and the bit error ratio (BER) is calculated.

\section{RESERVOIR COMPUTING}

$\mathrm{RC}$ is an option for the equalizer of Fig. 1. It is a highlyredundant recurrent $\mathrm{NN}$ (RNN) where only the output weights are trained, which simplifies the training process. Moreover, the recurrent connections allow the $\mathrm{NN}$ to track time-variant impairments, such as CD. These two characteristics make RC attractive for channel equalization.

The framework of RC was proposed in [26] to unify RNN with the core idea of adapting only part of the weights, while the others are defined randomly and kept constant. This concept was introduced by Jaeger with echo state networks (ESN) [22] and Mass with the liquid state machine (LSM) [23], independently. In principle, training all the weights of a RNN could potentially outperformance RC algorithms, 
however, they are hard to train with traditional methods based on gradient-descent. The convergence is also not guaranteed, and the calculation of the weights can be computationally expensive by requiring long training periods [27], [28].

The use of RC for an optical IM/DD channel equalization was first proposed in [16] with an implementation of a single nonlinear node with delayed feedback, which is called a time-delayed photonic reservoir. They showed experimentally the equalization of a 4-pulse amplitude modulated (PAM) signal focusing on Kerr nonlinearities. In [17] a linear passive reservoir with a second-order nonlinearity only at the output layer is used to equalize an OOK signal after fiber propagation by focusing on the impact of memory effect due to CD. In [18] the time-delayed photonic reservoir is compared with the Kramers-Kronig receiver. In [20] we showed the potential a digital RC implementation assisted by an optical preprocessing stage based on filtering. The potential of RC for equalization can also be extended outside optical communication, as in [29]. Here the authors showed similar performance of the RC compared to the Volterra for a nonlinear wireless channel.

The implementation of photonic RC has a strong potential for integration, which can be used for general computation in a non-von Neumann computer architecture. In [30] the authors demonstrated the first optoelectronic implementation of RC for pattern recognition with the experimental work demonstrated in [31]. Several applications can be found for solving spoken digit classification [30], [32], image classification [33], and nonlinear system prediction [34], [35]. For further details, an overview can be found in [36], [37], [38].

\section{A. RC implementation}

Fig. 2 shows the RC architecture considered in this work. It consists of an input layer, the reservoir, and the output layer. The number of inputs in the first layer depends on the application. Specifically for this work, Fig. 2 shows four inputs (plus bias) in the input layer, which come from the received signal spectral slices in which the time samples from different PDs are acquired in parallel. This means that over consecutive time samples all the signals are processed at the same time from different spectral slices. In other words, we have used a single input per signal received by a PD (4 input nodes) and a single bias (bias node)- this is to highlight the intrinsic characteristic of tracking time-variant impairments of the RC. The weights connecting the input layer to the reservoir $\left(W^{i n}\right)$ are drawn from a uniform distribution $\mathcal{U}(-1,1)$. The number of neurons in the reservoir is analyzed in the results section. When it is not analyzed the number is set to 500 neurons. In [20], we have shown that increasing the number of neurons in the reservoir also increases the memory of the system. The greater the memory required, the greater the number of neurons needed. The probability of interconnections of neurons in the reservoir is drawn from a binary distribution. Additionally, the probability of no interconnection corresponds to $98 \%$ of sparsity. Those non-zeros weights $\left(W^{r e s}\right)$ are then drawn from a standard normal distribution $\mathcal{N}(0,1)$ and kept fixed. The activation function is defined as a hyperbolic tangent. The output layer has 1 neuron with a linear activation function and has connections from the input layer $\left(W_{i n}^{\text {out }}\right)$ and from the reservoir $\left(W_{r e s}^{\text {out }}\right)$. These are the only weights that are trained through linear regression.

Equation (1) shows the nonlinear dynamic system that describes the evolution of the reservoir states $(\mathbf{x}[n])$.

$$
\begin{aligned}
\mathbf{x}[n]=\alpha \cdot \tanh \left(\mathbf{W}^{i n} \cdot \mathbf{u}[n]+\mathbf{W}^{r e s} \cdot \mathbf{x}[n-1]\right)+ \\
(1-\alpha) \cdot \mathbf{x}[n-1],
\end{aligned}
$$

where the leaking rate $\alpha$ is set to 0.9 and the input neurons are defined by $u[n]$. At the reservoir's output, a linear regression that minimizes the square error of the desired signal and $x[n]$ is applied yielding the network's readout $\mathbf{y}[n]$. A pseudocode of the implemented algorithm can be found in [20].

\section{EXPERIMENTAL SETUP}

The experimental setup is designed to compare a single carrier transmission with the optoelectronic receiver. The corresponding receiver structures for these two schemes are shown in the figures Fig. 3a and Fig. 3b, respectively.

At the transmitter, a random binary sequence at $32 \mathrm{GBd}$ with OOK is generated and shaped by a RRC filter (rolloff equal to 0.1 ) at 2 samples per symbol (sps). The resulted signal is resampled to the sampling rate of the digital-to-analog converter (DAC) operating at $88 \mathrm{GSa} / \mathrm{s}$. A Mach-Zehnder modulator (MZM) is then used to modulate the signal with the bias set at the quadrature point. The resulting optical signal is propagated through a length of single mode fiber (SMF) and amplified by an erbium-doped fiber amplifier (EDFA), irrespective of the equalizer used.

At the single carrier receiver (Fig. 3a), the wavelength selective switch (WSS) is set to a broadband filter, removing the out-of-band noise. The signal is then detected by one PD with a $3-\mathrm{dB}$ electrical bandwidth of $\approx 40 \mathrm{GHz}$.

At the optoelectronic receiver (Fig. 3b), the signal is filtered by a WSS with an optical filter as shown in Fig. 5a. The filter's bandwidth choice is based on our numerical analyses in [20] and briefly mentioned in sec. II. The filters are designed as a second-order Gaussian filter with a 3-dB bandwidth of $16 \mathrm{GHz}$. With $8 \mathrm{GHz}$ spacing of the spectral slices, this results in an overlap of about $8 \mathrm{GHz}$, as it is illustrated by the four colored curves in 5a. The signal is then detected independently by four PDs (same bandwidth as the single PD case).

After detection, irrespective of the receiver, the signal is digitally sampled by a real-time scope with $80 \mathrm{Gsa} / \mathrm{s}$ and $33 \mathrm{GHz}$ of electrical bandwidth. The received signal is then processed offline, with an low-pass filter, an equalizer, and a hard decision (DEC). Specifically, three different equalizers are tested independently for each receiver. For the single carrier receiver (Fig. 3a), a feedforward equalizer (FFE), RC, and a feedforward Neural Network (FNN) with hyperbolic tangent (FNN (tanh)) as the activation function are analyzed. For the optoelectronic receiver (Fig. 3b), FNN (tanh), RC and a FNN with linear activation function (FNN (linear)) are analyzed. The choice of using the FNN (linear) in the optoelectroinc receiver is to investigate the difference between 


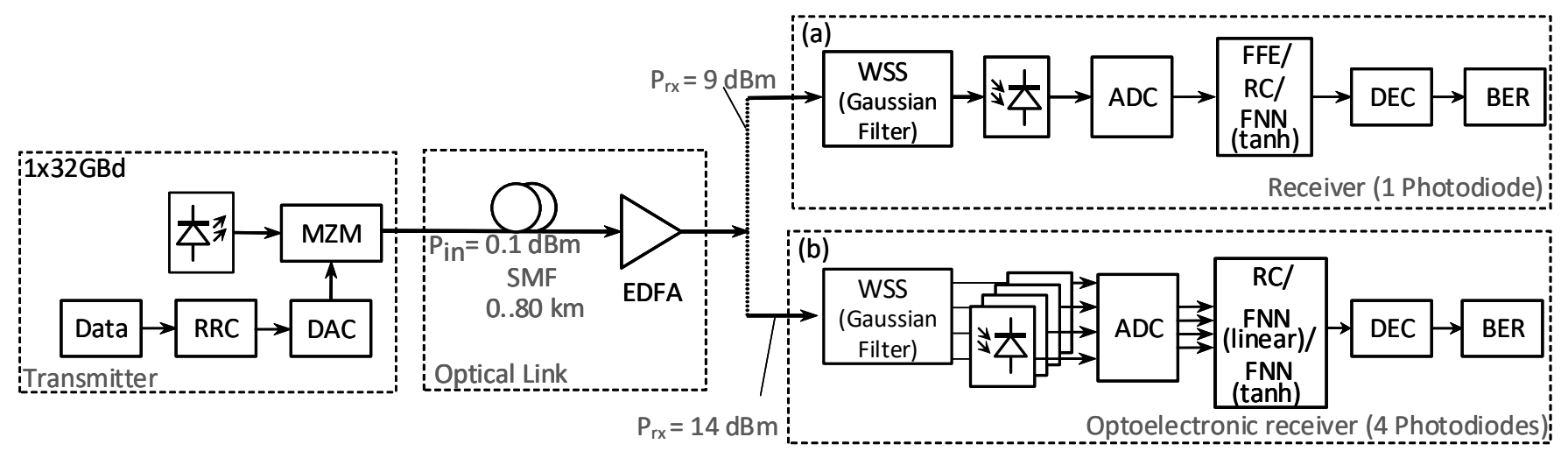

Fig. 3. Experimental setup. (a) single photodetector receiver. (b) optoelectronic receiver (4 photodetector).

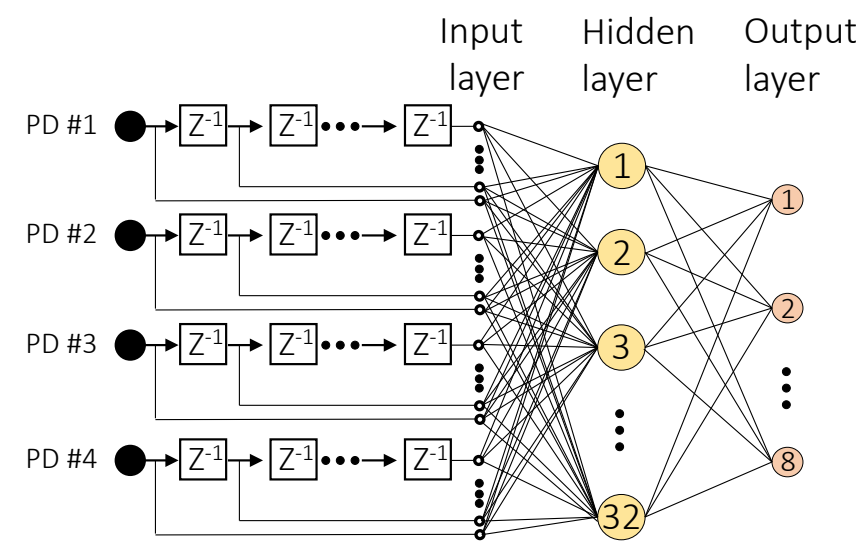

Fig. 4. Time-delay neural network structure. For the case with a single photodetector (PD) scenario, only one of the time delay structure is used.
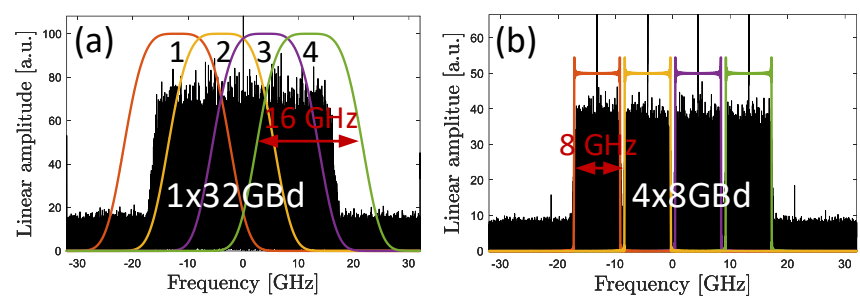

Fig. 5. Relative position of the optical filters. (a) 4 optical Gaussian filter for slicing a $32 \mathrm{GBd}$ OOK signal transmission. (b) 4 optical rectangular filter to separate each $8 \mathrm{GBd}$ subcarrier.

a linear and nonlinear equalizer with similar topology. Finally, the bit errors are counted, from that BER is evaluated.

The FFE is implemented with 32 taps updated by a least mean square algorithm at 2 sps. Fig. 4 shows the layout of the FNN. It is a 1 hidden-layer time-delay neural network equalizer with 32 neurons in the hidden layer and 8 linear neurons in the output layer which represents 1 symbol at 8 sps. The output is downsampled before the BER is counted. In contrast to the RC, the channel memory needs to be present at the FNN's input layer. Therefore an input temporal window is necessary. The temporal width of the window considered in this work is 5 symbols per PD with a window sliding of 1 symbol at a time. More specifically, in the case of 4 PDs, we are using 160 input samples (at 8 sps) plus 4 independent bias in the input layer. The central symbol is the one being equalized. This temporal width was chosen roughly equal to the spreading of a symbol pulse by the maximum chromatic dispersion over $80 \mathrm{~km}$. The LevenbergMarquardt backpropagation algorithm is used to update the weights at $8 \mathrm{sps}$ with $5 \%$ of the total data used as training, which is 79232 samples. After training, the weights are kept constant. The RC is implemented as discussed in section III with the same number of sps and training data as the FNN, but operating with 1 input neuron per PD plus bias. For all algorithms, the upsample to 8 sps was performed off-line as part of the receiver DSP chain. After using $5 \%$ of the total data as training, for all algorithms analyzed in this work, the taps were kept constant for the rest of the data. For real-time analyses or a longer sequence of data, a re-training will be necessary as time drift is present due to mismatching of the clock from the DAC and ADC. Alternatively, a time estimation algorithm can also be added to the DSP chain, however, this would increase the complexity of the receiver.

\section{EXPERIMENTAL RESULTS}

Fig. 6 shows a comparison of the performance in BER as a function of transmission reach, using the single carrier receiver (Fig. 3a) and optoelectronic receiver (Fig. 3b) for

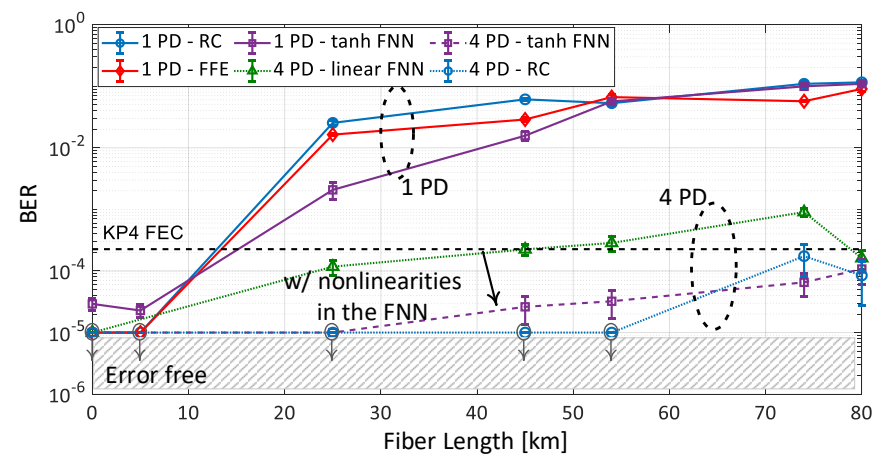

Fig. 6. Experimental comparison between single carrier transmission and optoelectronic receiver for $32 \mathrm{GBd}$ OOK signal considering different equalizers. 
different equalizers. The BER values are calculated over 200k symbols and are averaged over 10 measurements to increase the statistical relevance of the results. Furthermore, in all the following result analyses, the BER equal to zero (no errors) was replaced to a floor value of $10^{-5}$. This region is called "error-free", and it is chosen to highlight all the fiber distances analyzed in the experiment and numerical setup.

The single PD receiver showed a BER below the KP4 forward error correction (FEC) threshold $\left(\mathrm{BER}=2.24 \cdot 10^{-4}[40]\right)$ for a back-to-back and $5 \mathrm{~km}$ fiber transmission, irrespective of the equalizer. These few kilometers reach are expected due to the power fading (spectral notches) in the electrical signal from the interaction between chromatic dispersion and square-law detection, which makes the equalization process challenging for IM/DD and high symbol rate system. Fig. 6 shows that the tanh-FNN- and RC- equalizers, together with the optical pre-processing, can improve the transmission reach to $80 \mathrm{~km}$ of SMF. Although both equalizers show similar performance, the differences in the training process, and the number of neurons in the input layer should be highlighted. The FNN needs to train all the weights present in its network and a higher number of inputs (160 input samples plus bias) to create an equalization window to mitigate the spreading of the signal over time due to group velocity dispersion of the channel. The RC, on the other hand, only needs to train the weights in the output layer with a simpler algorithm and its memory comes from the feedback connections and depends on the reservoir properties. Because of that, only 1 sample per PD is necessary for the input layer of RC to mitigate the intersymbol interference. The linear FNN equalizer is also investigated to compare the performance of the two previously discussed nonlinear equalizers to a linear equalizer. In this case, changing only the activation function of the FNN (keeping the same number of neurons) showed a halving of the transmission reach compared to the tanh-FNN and RC equalizers.

Note, in the experiment the link loss does not scale strictly linearly with link length because different fiber spools with changing connector losses are used. Hence BER, which depends not only on CD but also on receiver OSNR determined by preamp EDFA input power, does not exhibit a monotonically increasing value with length, as can be seen in Fig. 6 .

Fig. 7 shows the impact of using less slices than the total

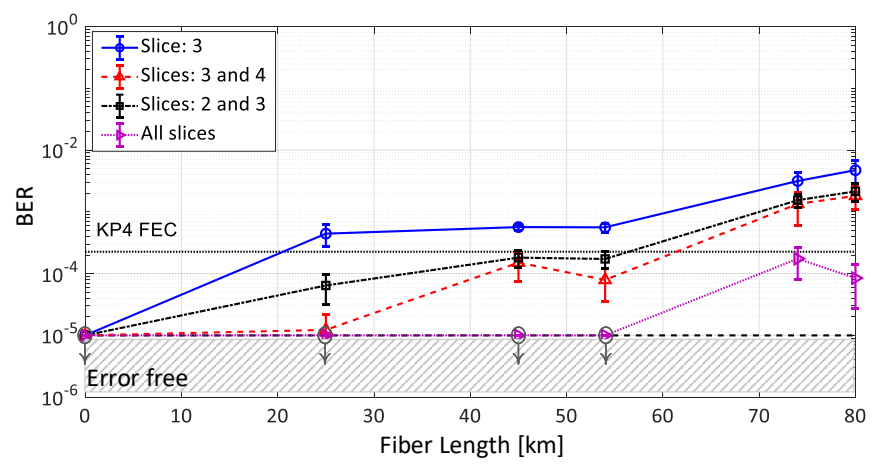

Fig. 7. Experimental results of using a reduced number of slices that are available in the receiver.

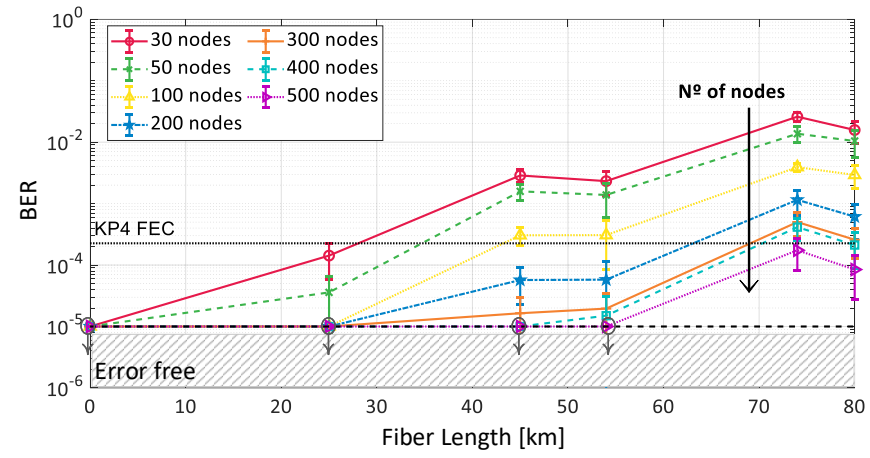

Fig. 8. Experimental results to compare the performance of different number of neurons in the reservoir.

number available for the optoelectronic receiver (Fig. 3b). Using only partial information to recover the transmitted information showed better performance compared to the $1 \mathrm{PD}$ receiver. This indicates the high impact of having notches in the electrical signal's spectrum. The scenario with only the slices 3 and 4 (indicated in Fig. 5a) can be compared to a single sideband receiver. As well, the slices 2 and 3 can be compared to a duobinary transmission. In both situations with reduced hardware effort, we achieve successful transmission up to $55 \mathrm{~km}$ with BER below the KP4 FEC limit.

Fig. 8 shows the impact of number of neurons in the RC for the optoelectronic receiver (Fig. 3b). Increasing the number of neurons improved the BER performance. This difference in performance might be related to the memory window available in the reservoir due to the echo state property [20]. Moreover, increasing the number of neurons also increases the diversity of signals in the RC's output [41]. In other words, the dimensionality space of the RC increases, therefore, improving the chances to have a better solution through linear transformation in the output layer to the desired signal.

\section{NUMERICAL SETUP}

The numerical setup is designed to compare the optoelectronic receiver with the subcarrier system. One of the main transmission reach gains of the optoelectronic systems comes from the spectral slicing process prior to PD detection. Reducing the signal's bandwidth also reduces the CD impact. In the SCM the symbol rate is already reduced in the transmitter. Comparing the optoelectronic system with this scheme may show the limitations of the proposed receiver. The numerical analyses were chosen to have a controlled environment in this investigation. Conventionally, SCM is multiplexed in the electronic domain and detected by a single photodetector (PD) [24], [25]. However, in this work, we have multiplexed the subcarriers optically and detected each independently. In this way, it is possible to re-use the same building block of the system in both situations for a fair comparison. Fig. 9 shows the numerical setup. The dashed blocks are used only when transmitting 4 independently subcarriers with lower symbol rate, keeping similar spectral efficiency as in the single carrier scenario. Fig. 9a, shows the single carrier receiver used as a reference, Fig. 9b the optoelectronic receiver, and Fig. 9c 
the subcarrier receiver, where each subcarrier is detected independently.

The numerical setup is divided in 2 sections to facilitate the comprehension. Section VI-1 described the single carrier transmission (without dashed blocks in the transmitter) received with a single PD (Fig. 9a) and the optoelectronic receiver (Fig. 9b). Section VI-2 described the subcarrier system (with dashed blocks in the transmitter) received independently by each PD (Fig. 9c).

1) Single carrier transmission: At the transmitter, a random stream of $2^{18}$ bits is generated and pulse shaped with RRC (roll-off equal to 0.1 ) at 2 sps, yielding a 32-GBd OOK signal. The signal is then upsampled to $8 \mathrm{sps}$ and modulated by the MZM's transfer function in the quadrature point. The DAC is not considered in the numerical analyses. This signal is then injected into the transmission channel. As the focus is on compensating for CD-induced ISI after detection, the transmission channel is modeled only as a linear lossless dispersive element with a dispersion parameter $D=16.4 \mathrm{ps} / \mathrm{nm} / \mathrm{km}$. An additive white Gaussian noise (AWGN) source is used to degrade the signal, e.g., simulating an optical pre-amplifier at the receiver, consistently with the experimental analysis. The signal-to-noise ratio (SNR) in the optical domain is kept fixed at $27 \mathrm{~dB}$. The SNR is defined according to the experimental optical SNR (OSNR) at back-2-back, with measured value of $34.58 \mathrm{~dB}$. The OSNR is mapped to SNR according to [39]. It is worth to highlight that we are using a single polarization signal in the system.

At the receiver, all PDs are modeled as noiseless squarelaw elements. For the case with 1 PD (Fig. 9a), the WSS is modeled as a Gaussian filter with the half-width halfmaximum (HWHM) bandwidth at $20 \mathrm{GHz}$. For the optoelectronic receiver (Fig. 9b), the WSS is modeled as 4 Gaussian filters with HWHM of $8 \mathrm{GHz}$ and its relative position in the signal's spectrum showed in Fig. 5a. The baseband central frequency of the filters are set to $-12 \mathrm{GHz},-4 \mathrm{GHz}, 4 \mathrm{GHz}$, $12 \mathrm{GHz}$. This means an spectrum overlap among the filter of $8 \mathrm{GHz}$. The ADC is not considered and the same algorithms for equalization mention in sec. IV are used here.

2) Subcarrier system: In the subcarrier system, the symbol rate is reduced to $8 \mathrm{GBd}$ and the same process mention in the transmitter of section VI-1 is reproduced here, independently, for 4 subcarriers. Additionally, each subcarrier is shifted to a central baseband frequency of $-13.2 \mathrm{GHz},-4.4 \mathrm{GHz}, 4.4 \mathrm{GHz}$, 13.2 GHz, to avoid the cross-talk among the carriers. The total bandwidth is also $35.2 \mathrm{GHz}$, as in the single-carrier case. After modulation, the signals are coupled together and transmitted to the SMF, as detailed in the previous section. AWGN source is added to keep the same SNR for each subcarrier as in the single carrier transmission system.

At the receiver (Fig. 9c), the WSS is modeled as 4 rectangular filters with HWHM of $4 \mathrm{GHz}$ and its relative position in the signal's spectrum showed in Fig. 5b. Therefore, avoiding cross-channel interference in the received signal. It should be noted that sharp filters are required in these analyses and they are a challenge to be obtained with the current technology. After filtering, the FFE described in section IV is used to process each signal independently, and the average BER of each subcarrier is evaluated.

\section{NUMERICAL ANALYSES}

The numerical results are also analysed in terms of transmission reach and BER at the KP4 FEC threshold. For each BER analysis, $5 \times 2^{18}$-symbol sequences are transmitted and the BER values are averaged over 5 independent transmissions to increase the statistical relevance of the results.

Fig. 10 shows the performance of a single carrier transmission for $32 \mathrm{GBd}$ and $8 \mathrm{GBd}$ OOK signal using the receiver with 1 PD (Fig. 3a). As expected, because of the square scaling factor related to the $\mathrm{CD}$, reducing the symbol rate transmission by a factor of 4 , increases the transmission reach by 16 times. The performance is approximate the same, irrespective of the equalizer used.

Fig. 11 shows the performance of a single carrier transmission for $32 \mathrm{GBd}$ OOK signal using the optoelectronic receiver with 4 PDs (Fig. 3b). After slicing the received signal, the time delay relate to the $\mathrm{CD}$ for each slice can be calculated deterministically concerning the signal's spectrum center. For example, considering the center of the first slice (indicated in Fig. 5a), the frequency difference to the center of the signal's spectrum is $-12 \mathrm{GHz}$. As well, considering the dispersion parameter $D=16.4 \mathrm{ps} / \mathrm{nm} / \mathrm{km}$ and the fiber transmission $L=80 \mathrm{~km}$, the time delay due to the CD can be calculated as $C D_{\text {delay }}=D * \Delta \lambda * L=126.17$ ps. The delay of each slice is then corrected before equalization by considering the sample rate of the system. The effects of this delay being applied (full-lines) or not (dashed-lines) is analyzed in Fig. 11 for the RC (circles) and the tanh-FNN (squares). The results show that for both equalizers the CD synchronization (CD sync), explained previously, increases the transmission reach by $80 \%$ for the RC and $60 \%$ for the tanh-FNN. Considering that the FNN and the RC are universal approximators (given the appropriate choice of number of neurons and training) [42], [43], both algorithms should be able to converge to a solution that would give the appropriate delays mentioned before. A probable reason why this is not happening is that these delays should be applied individually for each slice. In other words, the connection from the input signal to the hidden layer (or reservoir), for each input, should converge to a transformation that matches the necessary individual delays mentioned before. Therefore, achieving a reduced error in the cost function of the equalizer. In this sense, these connections would be working as interpolators. Changing the topology of these connections to act as interpolators with an initial condition close to the desired value would facilitate the convergence of the FNN. In the case of the RC, because the input weights are not trained, they would need to be defined deterministically. For the case of mitigation of $\mathrm{CD}$ in a short reach system using the optoelectronic receiver, this is not necessary since the delay is already known and only depends on the choice of the optical filters and symbol rate of the system.

Fig. 12 shows the performance of a single carrier transmission for $32 \mathrm{GBd}$ OOK signal using the optoelectronic receiver with 4 PDs (Fig. 3b) and the subcarrier system for $8 \mathrm{GBd}$ OOK signal (Fig. 3c). The results show an improvement of $40 \mathrm{~km}$ in 


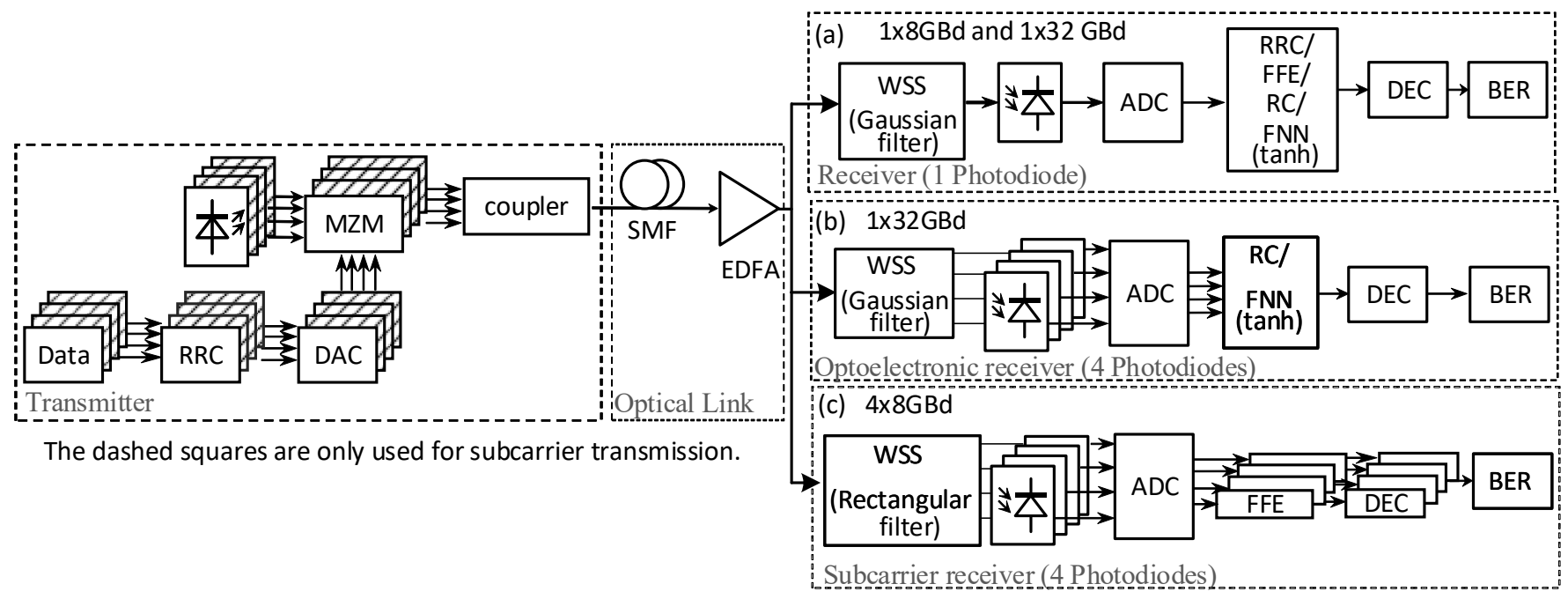

Fig. 9. Numerical setup. (a) single photodetector receiver. (b) optoelectronic receiver (4 photodetector). (c) subcarrier receiver (4 photodetector).

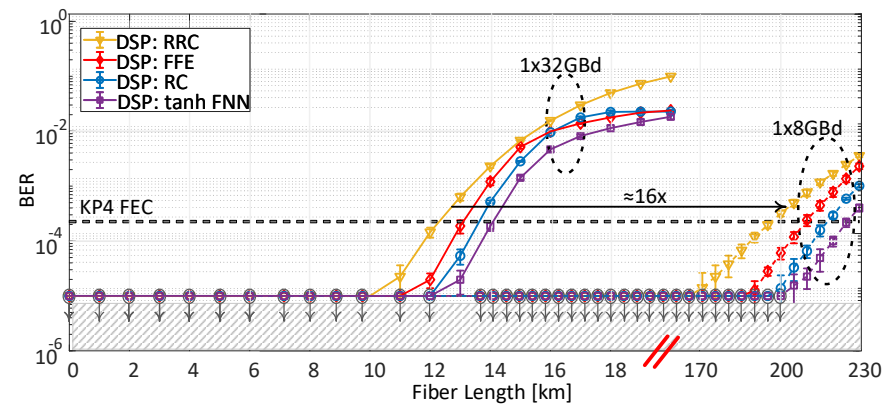

Fig. 10. Numerical analyses of the impact of chromatic dispersion on single carrier transmission considering different equalizers for $32 \mathrm{GBd}$ and $8 \mathrm{GBd}$ OOK signals.

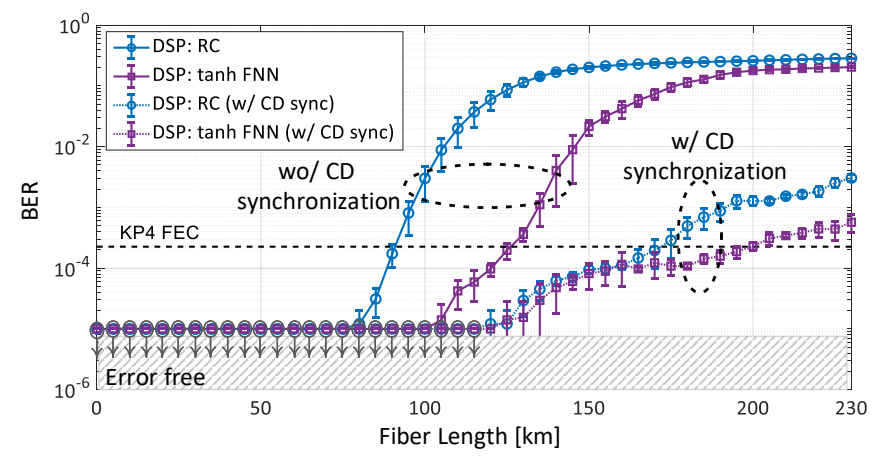

Fig. 11. Numerical analyses of the impact of slices synchronization on the optoelectronic receiver for single carrier transmission of $32 \mathrm{GBd}$ OOK signal.

the subcarrier system receiver compared to the optoelectronic system. It is worth to highlight that in the subcarrier system it requires a sharp optical or digital filter in the receiver to split the subcarriers without cross-talk. A more realistic scenario would show either a penalty in spectral efficiency or performance.

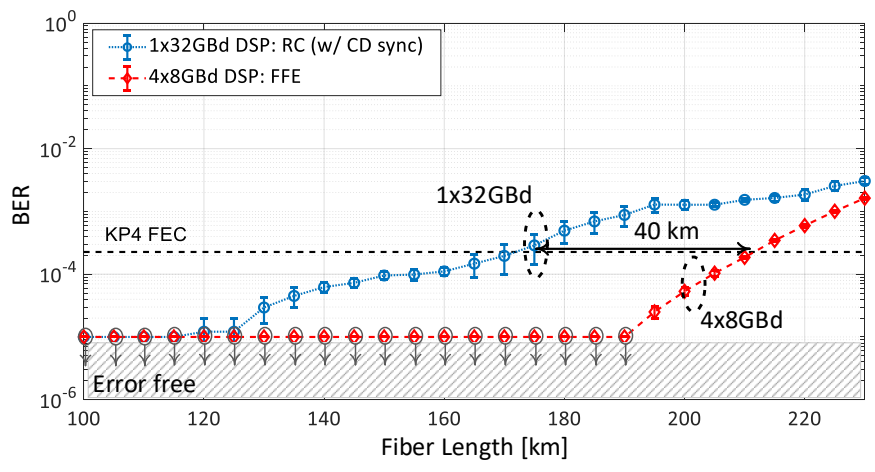

Fig. 12. Numerical comparison between optoelectronic receiver with slice synchronization $(1 \times 32 \mathrm{GBd})$ and subcarrier system $(4 \times 8 \mathrm{GBd})$.

\section{CONCLUSION}

We showed experimentally an optoelectronic receiver for a transmission with $32 \mathrm{GBd}$ OOK over $80 \mathrm{~km}$ of SMF in a DD system. The proposed optoelectronic receiver splits the signal into spectral slices which are then detected separately by PDs. Processing of the directly detected spectral slices by different options of $\mathrm{NN}$ after $\mathrm{ADC}$ showed that $\mathrm{RC}$ equalizer and FNN exhibit similar performance. However, we highlighted the difference in the training process and the high number of inputs nodes for the FNN to mitigate the intersymbol interference from the $\mathrm{CD}$. As mentioned, the simpler and faster training process of the $\mathrm{RC}$ can be an advantage considering that the system will require a periodic re-training. We also compared the results by using a NN with a linear activation function, probing the gain by the nonlinear equalizer for the optoelectronic receiver. Considering the nonlinear equalization with the optoelectronic receiver achieved $80 \mathrm{~km}$ of SMF, the use of linear activation function with the same number of neurons reduced the transmission reach by almost half, $\approx$ $45 \mathrm{~km}$. We also evaluate the performance of using only a selected number of slices (2 PDs) than the total available in the receiver (4 PDs). Using only partial information showed a 
reduction in the transmission reach. However, with the reduced hardware complexity, a transmission reach of $\approx 55 \mathrm{~km}$ below KP4 is still demonstrated. Additionally, we showed numerical analyses for the optoelectronic system considering a fixed time delay to correct the $\mathrm{CD}$ for each slice in the transmitter. Using this synchronization before equalizing the signal showed an $80 \%$ increase in the transmission reach for the RC and $60 \%$ for the FNN. Finally, we compared the 32 GBd OOK signal detected by an optoelectronic receiver with an $8 \mathrm{GBd}$ OOK signal in a subcarrier system. The analyses showed an increase of $\approx 40 \mathrm{~km}$ in transmission reach for the subcarrier system. As the required hardware is different for each situation, the tradeoff between performance and complexity should be analyzed for each situation.

\section{ACKNOWLEDGMENT}

This project has received funding from the European Union's Horizon 2020 research and innovation programme under the Marie Sklodowska-Curie grant agreement No 766115, the European Research Council through the ERCCoG FRECOM project (grant No 771878) and VYI OPTIC-AI project (grant No 29344).

\section{REFERENCES}

[1] J. K. Perin, A. Shastri, and J. M. Kahn, "Data center links beyond 100 Gbit/s per wavelength", Optical Fiber Technology, 44, pp. 69-85, 2018

[2] J. P. Turkiewicz, "Cost-effective $\mathrm{n} \times 25 \mathrm{Gbit} / \mathrm{s}$ DWDM transmission in the $1310 \mathrm{~nm}$ wavelength domain", Opt. Fiber Technol., 17(3), 69-85, 2011

[3] L. Grüner-Nielsen, S. N. Knudsen, B. Edvold, T. Veng, D. Magnussen, C. C. Larsen, and H. Damsgaard, Hans, "Dispersion compensating fibers", Optical Fiber Technology, 6(2), pp. 164-180, 2000.

[4] N. M. Litchinitser, B. J. Eggleton, and D. B. Patterson, "Fiber Bragg gratings for dispersion compensation in transmission: theoretical model and design criteria for nearly ideal pulse recompression", Journal of Lightwave Technology, 15(8), pp. 1303-1313, 1997.

[5] O. Agazzi, M. R. Hueda, H. S. Carrer, and E. D. Crivelli, "Maximumlikelihood sequence estimation in dispersive optical channels", Journal of Lightwave Technology, 23(2), pp. 749-763, 2005.

[6] M. Rubsamen, J. M. Gene, P. J. Winzer, and R.-J. Essiambre, "ISI mitigation capability of MLSE direct-detection receivers", IEEE Photonics Technology Letters, 20(8) pp. 656-658, 2008.

[7] G. O. Glentis, K. Georgoulakis, and C. Matrakidis, "Performance evaluation of Decision Feedback Equalizers in fiber communication links", 2014 6th International Symposium on Communications, Control and Signal Processing (ISCCSP), 2014.

[8] J. Jignesh, T. Eriksson, M. Chagnon, B. Corcoran, A. J. Lowery, F. Buchali, and H. Bülow, "Transmitter-side Volterra filtering for increased dispersion tolerance in 56 Gbaud PAM-4 systems", in Proc. Optical Fiber Communication Conference (OFC), San Diego, CA, USA, March 2018, paper M2C.6.

[9] Q. Hu, M. Chagnon, K. Schuh, F. Buchali, and H. Bülow, "High Data Rate Tomlinson-Harashima precoding-Based Pam Transmission", in Proc. European Conference on Optical Communication (ECOC), Dublin, Ireland, September 2019.

[10] A. Mecozzi, C. Antonelli, M. Shtaif, "Kramers-Kronig coherent receiver", Optica, 3(11), pp. 1220-1227, 2006.

[11] S. Gaiarin, X. Pang, O. Ozolins, R. T. Jones, E. P. da Silva, R. Schatz, U. Westergren, S. Popov, G. Jacobsen, and D. Zibar "High speed PAM-8 optical interconnects with digital equalization based on neural network". in Proc. Asia Communications and Photonics Conference (ACP), Wuhan, China, November 2016, Paper AS1C-1.

[12] M. Chagnon, B. Karanov, and L. Schmalen, "Experimental demonstration of a dispersion tolerant end-to-end deep learning-based imdd transmission system", in Proc. European Conference on Optical Communication (ECOC), Roma, Italy, September 2018. Paper Tu4F.6.

[13] B. Karanov, D. Lavery, P. Bayvel, and L. Schmalen, "End-to-End Optimized Transmission over Dispersive Intensity-Modulated Channels Using Bidirectional Recurrent Neural Networks", Optics Express, 27(14), pp. 19650-19663, 2019.
[14] J. Estaran, R. Rios-Müller, R M. A. Mestre, F. Jorge, H. Mardoyan, A. Konczykowska, J.-Y. Dupuy, and S. Bigo, "Artificial neural networks for linear and non-linear impairment mitigation in high-baudrate IM/DD systems", in Proc. European Conference on Optical Communication (ECOC), Düsseldorf, Germany, September 2016.

[15] M. Secondini, E. Forestieri, and G. Prati, "Adaptive Minimum MSE Controlled PLC Optical Equalizer for Chromatic Dispersion Compensation", Journal of lightwave technology, 21(10), pp. 2322, 2003.

[16] A. Argyris, J. Bueno, and I. Fischer, "Photonic machine learning implementation for signal recovery in optical communications", Scientific Reports, 8(1) pp. 8487, 2018.

[17] A. Katumba, X. Yin; J. Dambre, and P. Bienstman, "A Neuromorphic Silicon Photonics Nonlinear Equalizer For Optical Communications With Intensity Modulation and Direct Detection", Journal of Lightwave Technology, 37(10), pp. 2232-2239, May 2019.

[18] S. Li, S. Ohlendorf, and S. Pachnicke, "100 km 56 GBd PAM-4 Transmission using Photonic Reservoir Computing", in Proc. European Conference on Optical Communication (ECOC), September 2019, Paper Tu.2.B.

[19] S. Ranzini, F. Da Ros, H. Bülow, and D. Zibar, "Tunable Optoelectronic Chromatic Dispersion Compensation Based on Machine Learning for Short-Reach Transmission", Applied Sciences, 9(20) pp. 4332, 2019.

[20] F. Da Ros, S. Ranzini, H. Bülow, and D. Zibar, "Reservoir-computing based equalization with optical pre-processing for short-reach optical transmission”, IEEE Journal of Selected Topics in Quantum Electronics, 26(5), pp. 1-12, 2020.

[21] S. Ranzini, R. Dischler, F. Da Ros, H. Bülow, and D. Zibar, "Experimental Demonstration of Optoelectronic Equalization for Short-reach Transmission with Reservoir Computing", in Proc. European Conference on Optical Communication (ECOC), Brussels, Belgium, September 2020. [accepted for publication: avaliable at arXiv:2010.06502]

[22] H. Jaeger, "The "echo state" approach to analysing and training recurrent neural networks". Technical Report GMD Report 148, German National Research Center for Information Technology, 2001.

[23] W. Maass, T. Natschläger, and H. Markram, "Real-time computing without stable states: A new framework for neural computation based on perturbations". Neural Computation, 14(11), pp. 2531-2560, 2002.

[24] P. A. Greenhalgh, R. D. Abel and P. A. Davies, "Optical prefiltering in subcarrier systems', Electronics Letters, 28(19), pp. 1850-1852, 1992.

[25] R. Hui, B. Zhu, R. Huang, C. T. Allen, K. R. Demarest, D. Richards, "Subcarrier multiplexing for high-speed optical transmission', Journal of lightwave technology, 20(3), pp. 417, 2002.

[26] B. Schrauwen, D. Verstraeten, and J. Van Campenhout, "An overview of reservoir computing: theory, applications and implementations". in Proc. 15th European Symposium on Artificial Neural Networks, pp. 471-482, 2007.

[27] M. Lukoševičius, and H. Jaeger, "Reservoir computing approaches to recurrent neural network training", Computer Science Review 3.3 (2009), pp. 127-149, 2009

[28] S. Haykin, "Neural Networks: A Comprehensive Foundation", 3rd ed. Prentice-Hall, 2007.

[29] M. Bauduin, S. Massar, and F. Horlin, "Non-linear satellite channel equalization based on a low complexity echo state network", in Proc. 2016 Annual Conference on Information Science and Systems (CISS), pp. 99-104, 2016.

[30] K. Vandoorne, W. Dierckx, B. Schrauwen, D. Verstraeten, R. Baets, P. Bienstman, and J. Van Campenhout, "Toward optical signal processing using Photonic Reservoir Computing", Optics Express, 16(15), pp. 11182-11192, 2008.

[31] K. Vandoorne, P. Mechet, T. Van Vaerenbergh, M. Fiers, G. Morthier, D. Verstraeten, B. Schrauwen, J. Dambre, and P. Bienstman, "Experimental demonstration of reservoir computing on a silicon photonics chip", Nature Communications, 5, pp. 3541, 2014

[32] D. Brunner, M.C. Soriano, C.R. Mirasso, and I. Fischer, "Parallel photonic information processing at gigabyte per second data rates using transient states," Nature Communications 4, pp. 1364, 2013.

[33] B. Shi, N. Calabretta, and R. Stabile "Deep Neural Network Through an InP SOA-Based Photonic Integrated Cross-Connect," IEEE Journal of Selected Topics in Quantum Electronics, 26(1), pp. 7701111, 2020.

[34] L. Appeltant, M.C. Soriano, G. Van der Sande, J. Danckaert, S. Massar, J. Dambre, B. Schrauwen, C.R. Mirasso, and I. Fischer, "Information processing using a single dynamical node as complex system," Nature Communications, 2, pp. 468, 2011.

[35] A. Röhm, and K. Lüdge, "Journal of Physics Communications," Journal of Physics Communications, 2, pp. 085007, 2018.

[36] D. Brunner, M.C. Soriano, G. Van der Sande, "Photonic Reservoir Computing", Walter de Gruyter GmbH \& Co KG, 1st ed. , 2019 
[37] G. Tanaka, T. Yamane, J. B. Héroux, R. Nakane, N. Kanazawa, S. Takeda, H. Numata, D. Nakano, and A. Hirose, "Recent advances in physical reservoir computing: A review", Neural Networks, 2019.

[38] L. De Marinis, C. Marco, C. Piero, and A. Nicola, "Photonic Neural Networks: a Survey", IEEE Access, accepted for publication, 2019.

[39] R. Essiambre, and R. W. Tkach, "Capacity trends and limits of optical communication networks", Proceedings of the IEEE, 100(5), pp. 10351055, 2012.

[40] E. Agrell, and M. Secondini, M. "Information-theoretic tools for optical communications engineers", In Proc. of the IEEE Photonics Conference, pp. 99-103, 2018.

[41] M. Lukoševičius, "A practical guide to applying echo state networks", Neural networks: Tricks of the trade, pp. 659-686, 2012.

[42] K. Hornik, M. Stinchcombe, and H. White, "Multilayer feedforward networks are universal approximator", Neural networks, 2(5), pp. 359366, 1989.

[43] L. Grigoryeva, and J-P. Ortega, "Echo state networks are universal", Neural networks, 108, pp. 495-508, 2018. 